\title{
Expanded motor and psychiatric phenotype in autosomal dominant Segawa syndrome due to GTP cyclohydrolase deficiency
}

\author{
J L K Van Hove, J Steyaert, G Matthijs, E Legius, P Theys, R Wevers, A Romstad, L B Møller, \\ K Hedrich, D Goriounov, N Blau, C Klein, P Casaer
}

J Neurol Neurosurg Psychiatry 2006;77:18-23. doi: 10.1136/jnnp.2004.051664

\begin{abstract}
See end of article for authors' affiliations .....................

Correspondence to: Johan L K Van Hove, University of Colorado Health Sciences Center at Fitzsimmons, Biochemical Genetics Laboratory, Mail stop 8313, PO Box 6511, Aurora, CO 80045-0511, USA; Johan.Vanhove@ uchsc.edu
\end{abstract}

Received 10 August 2004 Revised version received 14 March 2005

Accepted 14 April 2005

\begin{abstract}
Background: Segawa syndrome due to GTP cyclohydrolase deficiency is an autosomal dominant disorder with variable expression, that is clinically characterised by L-dopa responsive, diurnally fluctuating dystonia and parkinsonian symptoms.

Objective: To delineate the neurological and psychiatric phenotype in all affected individuals of three extended families.

Methods: GTP cyclohydrolase deficiency was documented by biochemical analyses, enzymatic measurements in fibroblasts, and molecular investigations. All affected individuals were examined neurologically, and psychiatric data were systematically reviewed.

Results: Eighteen affected patients from three families with proven GTP cyclohydrolase deficiency were identified. Eight patients presenting at less than 20 years of age had typical motor symptoms of dystonia with diurnal variation. Five family members had late-presenting mild dopa-responsive symptoms of rigidity, frequent falls, and tendonitis. Among mutation carriers older than 20 years of age, major depressive disorder, often recurrent, and obsessive-compulsive disorder were strikingly more frequent than observed in the general population. Patients responded well to medication increasing serotonergic neurotransmission and to L-dopa substitution. Sleep disorders including difficulty in sleep onset and maintenance, excessive sleepiness, and frequent disturbing nightmares were present in $55 \%$ of patients. Conclusion: Physicians should be aware of this expanded phenotype in affected members of families with GTP cyclohydrolase deficiency.
\end{abstract}

S egawa syndrome or dopa responsive dystonia is a clinically defined movement disorder that typically presents in childhood with dystonia. ${ }^{12}$ Dystonia usually starts in the lower limbs with reduced ambulation, and is often characterised by a typical diurnal fluctuation, improving with sleep and worsening during the day. Other presenting symptoms include tremor, parkinsonism, focal dystonia, and spastic paraparesis and hyperreflexia. ${ }^{2}{ }^{3}$

Segawa syndrome is usually inherited as an autosomal dominant condition with variable penetrance, but autosomal recessive forms have also been described. Most frequently, it is caused by a mutation in the GCHI gene coding for GTP cyclohydrolase (OMIM 128230), the first and rate limiting enzyme in the biosynthesis of tetrahydrobiopterin. ${ }^{2}$ Tetrahydrobiopterin is the co-factor for the enzymes phenylalanine hydroxylase, tyrosine hydroxylase, tryptophan hydroxylase, and NO synthase. ${ }^{4}$ The reduced activity of tyrosine hydroxylase leads to decreased dopamine production, which is implicated in the movement disorder.

The reduced activity of tryptophan hydroxylase that results from tetrahydrobiopterin deficiency can result in reduced serotonin synthesis. Serotonin has been implicated in mood disorders such as depression, obsessive-compulsive disorders, and anxiety. However, depressive symptoms have been reported only in a single family with GTP cyclohydrolase deficiency. ${ }^{5}$ Dopamine and serotonin can be assessed in the cerebrospinal fluid (CSF) via their metabolites homovanillic acid (HVA) and 5-hydroxyindolacetic acid (5-HIAA). ${ }^{4}$ Dopamine is also the precursor of noradrenaline, which can be evaluated in the CSF via its metabolite 3-methoxy-4hydroxyphenylglycol (MHPG). Serotonin deficiency can be treated by providing the precursor, 5-hydroxytryptophan, which bypasses the metabolic block, or by using selective serotonin reuptake inhibitors. Melatonin is synthesised in the pineal gland from serotonin via $\mathrm{N}$-acetylserotonin, and is implicated in the onset and persistence of sleep. Reduced phenylalanine hydroxylase activity leads to elevated phenylalanine/tyrosine levels only after loading. This phenomenon provides the basis for the phenylalanine loading test. ${ }^{6}$ The physiologic and symptomatic implications of reduced brain NO synthase activity have not yet been defined, but low CSF nitrite and nitrate levels have been documented in patients with tetrahydrobiopterin deficiency. ${ }^{7}$

In this study we report clinical, biochemical, and molecular characteristics of 18 affected patients with different degrees of motor impairment from three families. These families were ascertained from probands that presented with typical Segawa symptoms. A surprisingly high incidence of psychiatric and sleep disturbances was observed.

\section{METHODS}

Twenty two members from three families participated in this study; 18 patients were considered affected by GTP cyclohydrolase deficiency based on biochemical and/or molecular testing. The study was approved by the local ethics committee and informed consent was obtained.

A biochemical geneticist and a paediatric neurologist examined all patients. A striking number of older patients in these families had been treated for depression, and some

Abbreviations: CSF, cerebrospinal fluid; DGGE, denaturing gradient gel electrophoresis; 5-HIAA, 5-hydroxyindolacetic acid; HVA, homovanillic acid; MHPG, 3-methoxy-4-hydroxyphenylglycol; MSLT, multiple sleep latency test 
family members complained of pronounced sleep disturbances. Therefore, psychiatric problems and sleep disturbances were systematically reviewed. As part of the clinical assessment, we obtained psychiatric data on 16 subjects. Fourteen of them underwent a structured interview by an experienced psychiatrist, using the Structured Clinical Interview for DSM-IV Axis I Disorders (SCID-I/P) (Version 2.0 ). In two subjects, psychiatric data were obtained from the treating psychiatrist or from the general practitioner.

Complaints of sleep disturbances were assessed using both the Pittsburgh Sleep Quality Index using scores $\geqslant 8$ as pathological, $^{89}$ and the Epworth Sleepiness Scale. ${ }^{10}$ Polysomnography was carried out on two consecutive nights using infrared video monitoring, four channel electroencephalogram, electromyogram at the chin and the tibialis anterior muscles, and respiratory measurements. Sleep stages were scored according to the Rechtschaffen and Kales criteria, ${ }^{11}$ and respiratory events were characterised according to the international recommendations. ${ }^{12}$ During daytime, the multiple sleep latency test (MSLT) was carried out at 9 am, 11 am, 3 pm, and 6 pm. ${ }^{13}$

CSF was obtained in the early morning in patients who were not taking L-dopa. The neurotransmitters HVA and 5-HIAA were measured in the 6th $\mathrm{ml}$ of CSF by HPLC with electrochemical detection. ${ }^{14}$ A phenylalanine loading test $(100 \mathrm{mg} / \mathrm{kg})$ was performed in the non-fasting condition according to Hyland et al, ${ }^{6}$ and the normal values confirmed in our laboratory in patients with various clinical presentations. The production of pterins was assayed in cytokine stimulated fibroblasts, followed by the measurement of GTP cyclohydrolase enzyme activity as described..$^{15}$ Analyses of urinary pterins and blood spot dihydropterine reductase were normal in all biochemically investigated patients (A.II-5, A.II-7, B.III-2, C.IV-1). Mutation analysis of the gene for GTP cyclohydrolase $(G C H 1)$ was completed using denaturing gradient gel electrophoresis (DGGE) followed by sequencing. ${ }^{16}$ In families A and B, deletion analysis of the GCHl gene was performed using a quantitative duplex PCR assay on the LightCycler (Roche Diagnostics, Mannheim, Germany) with an internal standard for each exon. ${ }^{17}$ For linkage analysis at the locus of the GCHI gene at position 53,290,900 to 53,360,000, CA repeats D14S984 at position 48,087,470, D14S978 at 49,903,270, and D14S276 at $53,673,110$ on chromosome 14 were amplified by PCR, using the primer sequences available in the Human Genome Database. ${ }^{18}$ Fragments were sized after electrophoresis on an ALF DNA sequencer (Amersham Biosciences, Uppsala, Sweden) and analysed using the Allelelinks software (Amersham Biosciences, Uppsala, Sweden). The lengths of the fragments are reported, although they have not been standardised against CEPH references. ${ }^{19}$ Statistical analyses used the Linkmap module of the Fastlink package, ${ }^{20}$ with distances between the markers D14S984 and D14S978 set at $2 \mathrm{cM}$, and between D14S978 and D14S276 at $4 \mathrm{cM}$.

\section{RESULTS}

\section{Biochemical and molecular analysis}

The pedigrees of the investigated families are shown in fig 1 .

In family A, patients II- 1 , III-3, and III-6 presented with typical Segawa syndrome. All affected individuals had a pathologically elevated phenylalanine/tyrosine ratio on the phenylalanine loading test (fig 2). Patients II-5 and II-7, who had presented with mild atypical motor symptoms, were biochemically characterised. Analysis of CSF monoamines showed normal values (table 1). However, the production of pterins in cytokine stimulated fibroblasts in these patients was clearly decreased (table 1) and GTP cyclohydrolase enzyme activity was deficient. DGGE analysis, sequencing of all exons and of 600 base pairs of the promoter as well as gene dosage studies did not identify a mutation in the GCHI gene. For linkage analysis, DNA from the family members II1, II-3, II-5, II-7, II-9, II-10, III-1, III-2, III-3, III-4, III-6, III-7, III- 8 , and III- 10 and their partners was available (fig 1). II-1, II-3, II-5, II-7, II-9, III-1, III-3, III-4, III-6, III-7, III-8, and III10 share an identical haplotype 239-256-94 at D14S984, D14S978, and D14S276. II-10 does not carry this haplotype. In III-2, the paternal haplotype is 239-256-90, which suggests that a crossover has occurred on the paternal chromosomes between D14S978 and D14S276. The three analysed markers are informative in all meioses, except for marker D14S276 in family members II-3 and II-7. Potential crossovers between D14S978 and D14S276 in the next meioses (III-3, III-4, III-6, III-7, and III-8) would thus not be identified. Thus, family members II-10 and III-2 were unaffected based on a normal phenylalanine loading test and absence of the disease associated haplotype at the GCHI locus. Multipoint linkage analysis in this family for Segawa syndrome using markers D14S984, D14S978, and D14S276 showed a multipoint LOD score of 3.51 at position D14S276.

In family B, patient III-2 presented with typical Segawa syndrome. A phenylalanine loading test showed pathological values of the phenylalanine/tyrosine ratio in individuals II- 1 , III-1, and III-2 (fig 2). Analysis of CSF monoamines in patient III-2 showed slightly decreased HVA and MHPG, and low-normal 5-HIAA (table 1). The production of pterins in cytokine stimulated fibroblasts and GTP cyclohydrolase enzyme activity was deficient (table 1). Mutation analysis of the GCHI gene by DGGE and sequencing of all exons did not identify a mutation. However, a heterozygous deletion of the entire GCHI gene was identified by gene dosage studies in individual II-l and her daughter III-2. There was no DNA available from patient III- 1 to test for the mutation.

In family C, patients II- 1 and IV-1 presented with typical Segawa syndrome. A phenylalanine loading test showed a pathological phenylalanine/tyrosine ratio in patient IV-1. This patient had decreased levels of HVA, MHPG, and 5-HIAA in her CSF (table 1). A heterozygous mutation p.Y75S (c.372A $>$ C) was identified in the GCHl gene in all three patients (II-1, III-1, and IV-1). A mutation involving this amino acid has been reported previously. ${ }^{21}$

\section{Clinical investigations}

Neurological examination (table 2) showed that most patients had symptoms of dystonia (56\%) and frequent presence of rigidity $(61 \%)$ and bradykinesia $(28 \%)$. In half of the patients, a marked diurnal fluctuation was present. Four subjects with positive biochemical signs and genetic findings were completely asymptomatic at ages 13, 20, 20, and 23 years. Hyperreflexia was frequently present, but other signs of pyramidal tract involvement were infrequent and cerebellar signs were always absent. Several patients reported an increased number of falls, even if they did not have notable dystonia and only minimal rigidity. Two patients were wheelchair bound. Surprisingly, five patients identified through family analyses complained of recurrent tendonitis, often requiring various orthopaedic therapies. These problems were usually associated with mild rigidity, and subjectively with stiffness and tiredness, but without dystonic symptoms. Symptoms usually appeared after more strenuous exercise and had been considered unrelated to the family history of Segawa syndrome. However, on low dose Ldopa/carbidopa therapy all tendonitis symptoms completely disappeared and patients reported less stiffness and tiredness. No patient was intellectually impaired.

The psychiatric assessment (table 3 ) showed that major depressive disorder occurred in $44 \%$ of all carriers $(50 \%$ of psychiatrically investigated carriers), and that recurrent major depressive disorder occurred in $28 \%$ of all carriers (31\% of psychiatrically investigated carriers). This is well above the population risk, where the lifetime prevalence of 


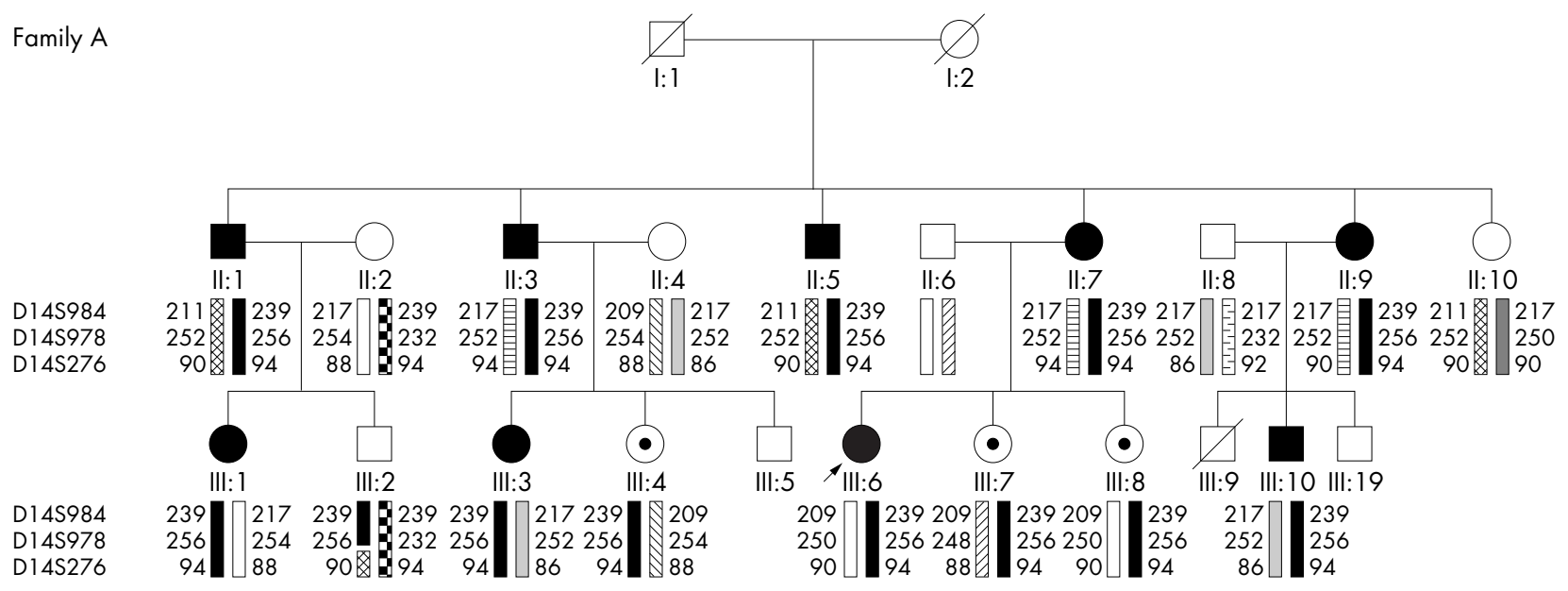

Family B
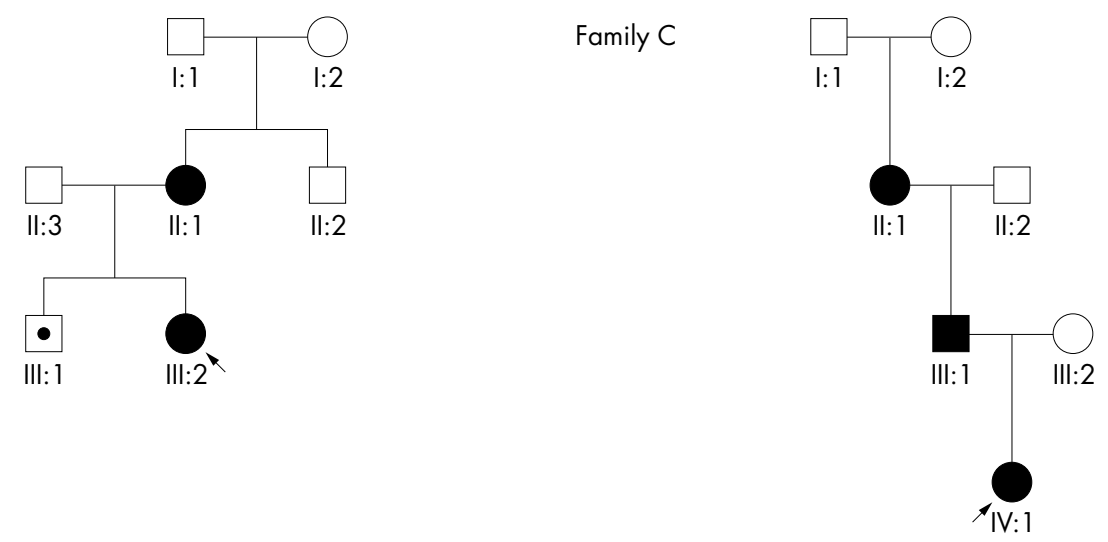

Figure 1 Family pedigrees. Filled symbols indicate those affected by GTP cyclohydrolase deficiency and symptomatic; a dot indicates those affected but asymptomatic. Linkage markers are indicated in family $A$.

major depressive disorder is estimated at $19 \%$ (SE 0.5) in the Netherlands ${ }^{22}$ and $16 \%\left(95 \%\right.$ CI 15.1 to 17.3 ) in the US. ${ }^{23} 24$ Major depressive disorder was observed in each of our three families with GTP cyclohydrolase deficiency. In nine out of ten patients, the depression was successfully treated with medication, which included serotonin reuptake inhibitors in six patients, a serotonin-noradrenaline reuptake inhibitor

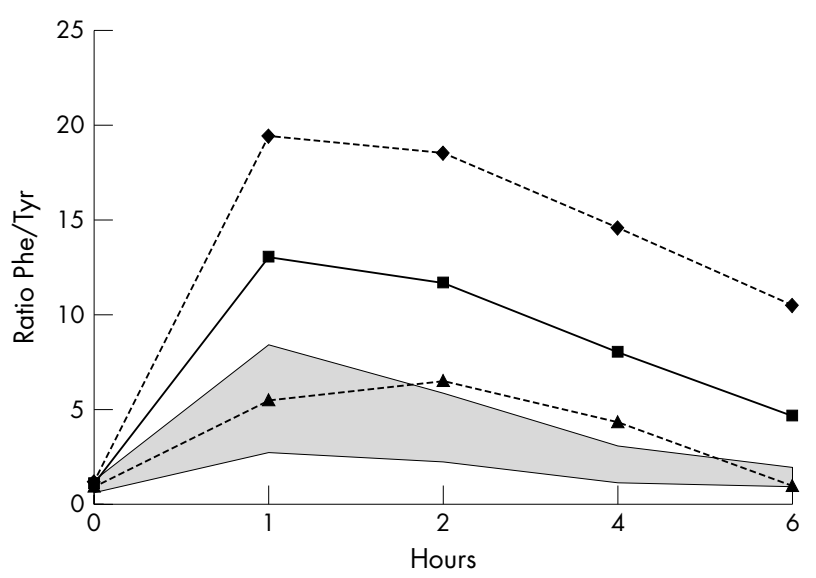

Figure 2 The phenylalanine loading test in 15 affected individuals. The shaded area indicates normal values according to Hyland et at and our own data. The minimum values $\boldsymbol{\Lambda}$, mean values $\boldsymbol{\square}$, and maximum values $\downarrow$ of all individual loading tests at each time point are given. and a serotonin agonist in one patient each, and 5hydroxytryptophan alone or a combination of these drugs in four patients. In at least two patients, the response to serotonin reuptake inhibitors was independent of the response to L-dopa. Obsessive-compulsive disorder was present in four patients $(22 \%)$, a frequency well above the population frequency $(0.5-3.5 \%))^{22}{ }^{25-28}$ All patients with obsessive-compulsive disorder had also had major depressive disorder and had responded, sometimes markedly, to serotonin-reuptake inhibitors. Anxiety disorders were present $(22 \%)$ at a frequency similar to the general population. ${ }^{22}$

Many patients complained of difficulty falling asleep and of sleep discontinuation. Some patients reportedly had less than $4 \mathrm{~h}$ of sleep each night. Two patients had a remarkably excessive need for sleep, requiring more than $10 \mathrm{~h}$ per night as an adult or falling asleep during the day if they slept less than this at night. Recurrent nightmares were frequently reported (22\%) even when not taking serotonergic medication. In patients A.II-5 and A.II-7, the Pittsburgh Sleep Quality Index was pathological at 15 and 13 prior to treatment; this improved to 11 and 5 after treatment with L-dopa (normal $<8$ ). These patients were further studied while taking this medication. The Epworth Sleepiness Scale revealed normal scores. Sleep polysomnographic studies, obtained on the second night of a two night polysomnography, were normal except for a reduction in REM sleep ( 5 and $9 \%$, control $10-28 \%$ ), which is a known medication side effect. ${ }^{29}$ The MSLT was also normal.

The onset of typical neurological dystonia symptoms differed from that of psychiatric symptoms (fig 3). The onset 
Table 1 Biochemical and enzymatic investigations

\begin{tabular}{|c|c|c|c|c|c|c|}
\hline & $\begin{array}{l}\text { HVA, nmol/I } \\
\text { (controls) }\end{array}$ & $\begin{array}{l}\text { MHPG, nmol/I } \\
\text { (controls) }\end{array}$ & $\begin{array}{l}\text { 5-HIAA, nmol/I } \\
\text { (controls) }\end{array}$ & $\begin{array}{l}\text { Neopterin (FB), } \\
\mathrm{pmol} / \mathrm{mg} \\
\text { protein (controls) }\end{array}$ & $\begin{array}{l}\text { Biopterin (FB), } \\
\text { pmol/mg } \\
\text { protein (controls) }\end{array}$ & $\begin{array}{l}\text { GTP-CH (FB), } \\
\mu \mathrm{U} / \mathrm{mg} \\
\text { protein (controls) }\end{array}$ \\
\hline A.II-5 & 347 (87-372) & NA & $194(58-190)$ & $24(18-98)$ & $98(154-303)$ & $0.83(1.4-6.5)$ \\
\hline A.II-7 & $99(87-372)$ & $36(29-64)$ & $67(58-190)$ & $18(18-98)$ & $50(154-303)$ & $0.43(1.4-6.5)$ \\
\hline B.III-2 & 75 (87-372) & 28 (29-64) & $66(58-190)$ & 10 (18-98) & 30 (154-303) & 0.39 (1.4-6.5) \\
\hline C.IV-1 & $195(330-668)$ & $29(32-68)$ & 81 (109-214) & NA & NA & NA \\
\hline
\end{tabular}

CSF levels of HVA (homovanillic acid), MHPG (3-methoxy-4-hydroxyphenylglycol), and 5-HIAA (5-hydroxyindolacetic acid). Fibroblast assays: Neopterin (FB): synthesis of neopterin in cytokine stimulated fibroblasts; Biopterin (FB): synthesis of biopterin in cytokine stimulated fibroblasts; GTP-CH (FB): GTP cyclohydrolase enzyme activity in fibroblasts. NA, not available. Control values for monoamines are age adjusted.

of neurological symptoms had a bimodal appearance. Nine patients had onset of typical dystonia symptoms in childhood $(<20$ years of age). A second group of five patients had onset of motor symptoms in adulthood at $>30$ years of age. These latter patients were all identified through family studies. Their symptoms consisted of the less typical complaints of rigidity with recurrent tendonitis responsive to low dose Ldopa. Psychiatric symptoms tended to have onset in adulthood with a peak incidence at 41-50 years of age, but could also have appeared in childhood.

Two patients presented first with psychiatric problems before developing motor symptoms. Three patients developed psychiatric symptoms after developing motor symptoms. Three patients developed psychiatric and motor symptoms around the same time. Five patients had motor symptoms but did not develop psychiatric symptoms.

Typical and atypical late onset symptoms appeared in the same family. In family A, patients II-1, III-3, and III-6 had a typical childhood onset complete Segawa syndrome, whereas other patients had a late onset atypical presentation; in family B, patient III-2 had a typical childhood onset Segawa syndrome, whereas patient II-l had an atypical late onset presentation. Patients with psychiatric symptoms sometimes had a severe motor syndrome, but often an atypical late onset presentation. The phenylalanine loading test did not distinguish between typical, atypical, or asymptomatic carriers (average phenylalanine/tyrosine ratio at $4 \mathrm{~h}$ : typical 7.1 , $\mathrm{n}=3$; atypical 7.4, $\mathrm{n}=7$; and asymptomatic 9.4, $\mathrm{n}=4$ ).

\section{DISCUSSION}

Affected individuals in all families were shown to have GTP cyclohydrolase deficiency by enzymatic and molecular analyses. A defect in the gene encoding GTP cyclohydrolase ( $G C H 1)$ was demonstrated in two families, a heterozygous gene deletion and a missense mutation, respectively, and can be assumed in the third family because all affected individuals share the same haplotype in the $\mathrm{GCHI}$ chromosomal region. In our laboratory, the phenylalanine loading test readily identified affected patients, with the time point at $4 \mathrm{~h}$ having the best diagnostic value (fig 2), but did not predict the extent of clinical symptoms. However, CSF neurotransmitter metabolite values, obtained in clearly affected patients, were more variable. Despite the consistent finding of reduced dopamine on histochemical staining in autopsied patients, ${ }^{30}$ similar variation of CSF metabolites has been observed and may reflect the difficulties of assessing a partial deficiency against a broad normal range in CSF. Decreased CSF biopterin levels have been described in GTP cyclohydrolase deficient patients, but this analysis was not available in this study.

Most patients with typical Segawa syndrome and all probands presented with typical symptoms of dystonia, frequently with noticeable diurnal variation as had been originally described. ${ }^{1}$ However, family investigations revealed many affected patients with less typical symptoms. In particular, the presentation with recurrent tendonitis and mild rigidity has not been described previously. The consistent response to L-dopa/carbidopa therapy suggests that these symptoms are indeed causally related. Both symptomatic presentations, childhood onset typical Segawa syndrome and late onset atypical presentations, occur in the same family in patients affected with the same mutation. This variability indicates that other factors, be they environmental or other modifying genes, influence the expression of symptoms. ${ }^{31}$

In this study, the frequency of major depressive disorder, particularly recurrent major depressive disorder, and of obsessive-compulsive disorders was clearly increased above the population frequency. However, this study may underestimate the frequency of psychiatric morbidity since several asymptomatic patients were less than 20 years of age, and psychiatric symptoms tended to have onset after 20 years of age (fig 3). Although the occurrence of major depressive disorder is significantly increased in the study group, a increased risk due to genetic factors independent of GTP cyclohydrolase deficiency cannot completely be excluded. However, this explanation is unlikely as no depressive disorders were reported in the non-carriers in the studied families, although these were not assessed as control subjects. Moreover, it is unlikely that an independent

Table 2 Neurological symptoms in GTP cyclohydrolase deficient patients

\begin{tabular}{|c|c|c|c|}
\hline Extrapyramidal, $78 \%$ & $14 / 18$ & Decreased functionality & \\
\hline Dystonia, total $56 \%$ & $10 / 18$ & Falls & $6 / 18$ \\
\hline Foot & $8 / 18$ & Wheelchair bound & $2 / 18$ \\
\hline Gait & $7 / 18$ & Cerebellar signs & $0 / 18$ \\
\hline Writer's cramp & $7 / 18$ & Pyramidal signs & \\
\hline Torticollis & $1 / 18$ & Reduced strength & $4 / 18$ \\
\hline Truncal flexion dystonia & $4 / 18$ & Spasticity & $2 / 18$ \\
\hline Hyperlordosis & $7 / 18$ & Babinski sign & $0 / 18$ \\
\hline Tremor & $5 / 18$ & Hyperreflexia & $6 / 18$ \\
\hline Rigidity & $11 / 18$ & Axial hypotonia & $3 / 18$ \\
\hline Tendonitis & $5 / 18$ & Diurnal fluctuation, $56 \%$ & $10 / 18$ \\
\hline Bradykinesia & $5 / 18$ & Asymptomatic, $22 \%$ & $4 / 18$ \\
\hline Tics & $1 / 18$ & & \\
\hline
\end{tabular}


Table 3 Psychiatric symptoms and sleep disturbances in GTP cyclohydrolase deficient patients affected with Segawa syndrome

\begin{tabular}{|c|c|c|c|c|}
\hline & \multirow[b]{2}{*}{ GCHI } & \multicolumn{2}{|l|}{ Present study } & \multirow[b]{2}{*}{ Population (range } \\
\hline & & $\begin{array}{l}\text { Psychiatrically } \\
\text { investigated, } n=16\end{array}$ & All, $n=18$ & \\
\hline MDD, total & 8 & $50 \%$ & $44 \%$ & $17 \%(15.1-17.3)$ \\
\hline$M D D$, recurrent & 5 & $31 \%$ & $28 \%$ & $9 \%$ \\
\hline$O C D$ & 4 & $25 \%$ & $22 \%$ & $1-3 \%(0.1-3.5)$ \\
\hline Anxiety disorders & 4 & $25 \%$ & $22 \%$ & $19.3 \%$ (SE 0.5) \\
\hline Psychiatric conditions (total number of subjects for MDD and OCD) & 8 & $50 \%$ & $44 \%$ & \\
\hline Problems in sleeping or sleep continuation & 7 & $43 \%$ & $39 \%$ & \\
\hline Excessive sleeping & 2 & $13 \%$ & $11 \%$ & \\
\hline Recurrent nightmares & 4 & $25 \%$ & $22 \%$ & \\
\hline Sleep disturbances (total number of subjects) & 10 & $62 \%$ & $55 \%$ & \\
\hline
\end{tabular}

increased risk would be found in three unrelated families. Increased frequency of major depressive disorder and anxiety was previously recognised in a single large family with GTP cyclohydrolase deficiency, ${ }^{5}$ and depression was reported in three other patients. ${ }^{32}{ }^{33}$ In that family, ${ }^{5}$ obsessive-compulsive disorder was also present in a single patient. In our study, anxiety was also observed in a number of patients but did not exceed the high population frequency. It is important to note that most patients with psychiatric morbidity were not yet aware that they had Segawa syndrome when their psychiatric morbidity developed. Therefore, psychiatric morbidity cannot be attributed to the stress of their knowing about their chronic neurological disorder. In the previously reported family $^{5}$ and in our three families, patients with psychiatric morbidity responded well to serotonin reuptake inhibitors or to other serotonin neurotransmission enhancing medication including supplementation with 5-hydroxytryptophan. In contrast to a previous report, ${ }^{33}$ we did not observe a reduction in the efficacy of L-dopa treatment on dystonia symptoms with these medications; nonetheless, caution is advisable as severe parkinsonism was seen with neuroleptic medications and with metoclopramide.

The role of disturbed dopamine metabolism in the pathogenesis of the motor dysfunction in Segawa syndrome has been well studied. ${ }^{34}$ Decreased striatal biopterin results in a reduction in the quantity and activity of tyrosine hydroxylase, enhancing the dopamine deficiency. ${ }^{35}$ In this condition, the functional consequences of impairment in the synthesis of the other neurotransmitters, serotonin and noradrenaline, have received less attention. Preclinical research and, in particular, clinical studies with serotonin reuptake inhibitors, strongly suggest that a deficit in serotonergic neurotransmission plays a key role in the pathogenesis of major depressive disorder. ${ }^{36-38}$ Whether a sole serotonergic dysfunction is sufficient to cause depression or

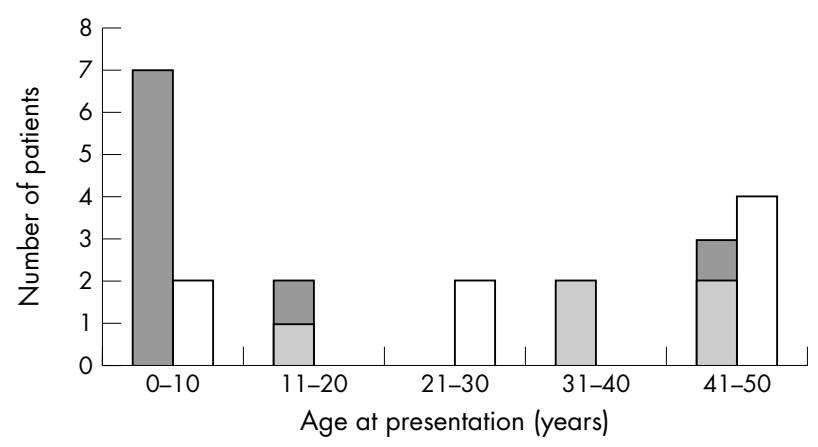

Figure 3 Age at onset of symptoms. Grey: neurological symptoms, of which dark grey severe neurological symptoms and light grey mild neurological symptoms (tendonitis and rigidity only); white: psychiatric symptoms. is a necessary risk factor remains unclear. Although the role of serotonin depletion in humans has not been studied, the impact of depletion of its precursor L-tryptophan has been investigated. L-tryptophan depletion only leads to depressed mood in patients who have a history of major depressive disorder, especially in patients with recently remitted major depressive disorder. ${ }^{39}$ We propose that similar biochemical consequences on serotonin levels result from either the depletion of tryptophan or from a genetic impairment of the synthetic enzyme in the serotonin biosynthetic pathway from tryptophan. This would imply that a specific vulnerability factor contributes to the expression of psychiatric symptoms in combination with the serotonin depletion. Modifier genes, or historic or environmental contributions, such as a previous depression, may affect this vulnerability. Consequently, patients lacking specific risk factors may only have the neurological symptoms of Segawa syndrome but not the psychiatric symptoms.

The deficit in the synthesis of L-dopa in Segawa syndrome theoretically also results in decreased noradrenaline, as is evidenced by somewhat decreased MHPG levels in CSF, and would be expected to be increased with L-dopa therapy. Noradrenergic deficiency, particularly in the locus coeruleus, has been hypothesised to contribute to depressive symptoms, ${ }^{40-42}$ and selective noradrenaline reuptake inhibitors are effective in its treatment. ${ }^{42}$ Several of our patients reported some improvement in mood following initiation of L-dopa therapy. The presence of a major depressive disorder in a single patient with tyrosine hydroxylase deficiency and very low MHPG levels (20 nmol/l, controls 29-64) (unpublished observation) also suggests a contribution of the noradrenergic neurotransmission. However, chronic L-dopa therapy did not prevent the occurrence of depressive symptoms in some patients who subsequently responded promptly to a serotonergic/noradrenergic reuptake blocker.

In conclusion, we would hypothesise that impairment of the synthesis of both serotonergic and noradrenergic neurotransmission may be related to the particularly high frequency of depression in this genetic condition. The patients have a well documented genetic impairment in the synthetic capacity of these neurotransmitters. The direct measurement of the metabolites of serotonin (5-HIAA) and of noradrenalin (MHPG) in CSF did not provide proof of a deficiency. However, analysis of CSF metabolites provides an indirect measurement at a distance of the presence of these neurotransmitters in brain with a wide normal range, and thus the measured low normal values do not disprove the possibility of a partial deficiency in the brain.

Sleep disturbances were very frequently reported in our adult Segawa patients and were sometimes the predominant symptoms. Previous studies documented reduced gross and twitch movements during sleep in Segawa patients, which improved under L-dopa treatment, ${ }^{132}$ and sleep disorders are frequent in patients with extrapyramidal disease. ${ }^{43}$ Both 
dopaminergic and serotonergic neurotransmission have been implicated in sleep mechanisms. Serotonergic raphe neurons have a crucial role in the initiation and maintenance of the sleep state. ${ }^{44}$ Further, serotonin is a precursor of melatonin, which is implicated in the initiation and persistence of sleep. Occasional patients reported improved sleep with melatonin intake.

Physicians caring for patients with Segawa syndrome should be aware of the development of late symptoms in adults affected with this disorder. Patients who are asymptomatic in childhood may develop dopa responsive atypical symptoms as adults. Further, patients are at increased risk for depression and obsessive-compulsive disorders. These disorders respond well to serotonergic neurotransmission enhancing medications, although monitoring for worsening of dystonia is advisable. ${ }^{35}$ Finally, sleep disturbances are very frequent in affected individuals. Finding such treatable conditions underscores the value of extensive biochemical and molecular testing in families with Segawa syndrome.

\section{ACKNOWLEDGEMENTS}

We thank M. De Becker, RN, for assistance in the biochemical investigations of the patients.

\section{Authors' affiliations}

J L K Van Hove, Department of Pediatrics, University of Colorado Health Sciences Center, Denver, CO, USA

J L K Van Hove, P Casaer, Department of Pediatrics, University Hospital Gasthuisberg, Catholic University of Leuven, Leuven, Belgium J Steyaert, Department of Child Psychiatry, University Hospital Gasthuisberg, Catholic University of Leuven, Leuven, Belgium G Matthiijs, E Legius, Department of Genetics, University Hospital Gasthuisberg, Catholic University of Leuven, Leuven, Belgium P Theys, Department of Neurology, University Hospital Gasthuisberg, Catholic University of Leuven, Leuven, Belgium

R Wevers, University Medical Center, Niimegen, The Netherlands

A Romstad, L B Møller, John F. Kennedy Institute, Glostrup, Denmark

K Hedrich, C Klein, Departments of Neurology and Human Genetics,

University of Lübeck, Lübeck, Germany

D Goriounov, N Blau, University Children's Hospital, Zürich, Switzerland

This work was supported in part by the Deutsche Forschungsgemeinschaft (KH and $\mathrm{CK}$ ) and by Swiss National Science Foundation grant no. 31-66953.01 (NB)

Competing interests: none declared

The patients described in this report gave their informed consent for their details to be published

\section{REFERENCES}

1 Segawa M, Hosaka A, Miyagawa F, et al. Hereditary progressive dystonia with marked diurnal fluctuation. Adv Neurol 1976;14:215-33.

2 Németh $\mathbf{A H}$. The genetics of primary dystonias and related disorders. Brain 2002; 125:695-721.

3 Bandmann O, Marsden CD, Wood NW. Atypical presentations of doparesponsive dystonia. Adv Neurol 1998;78:283-90.

4 Blau N, Bonafé L, Thöny B. Tetrahydrobiopterin deficiencies without hyperphenylalaninemia: diagnosis and genetics of DOPA-responsive dystonia and sepiapterin reductase deficiency. Mol Genet Metab 2001;74:172-85.

5 Hahn H, Trant MR, Brownstein MJ, et al. Neurologic and psychiatric manifestations in a family with a mutation in exon 2 of the guanosine triphosphate-cyclohydrolase gene. Arch Neurol 2001;58:749-55.

6 Hyland K, Freiburg JS, Wilson WG, et al. Oral phenylalanine loading in dopa-responsive dystonia: a possible diagnostic test. Neurology 1997;48:1290-7.

7 Zorzi G, Thöny B, Blau N. Reduced nitric oxide metabolites in CSF of patients with tetrahydrobiopterin deficiency. J Neurochem 2002;80:362-4.

8 Buysse DJ, Reynolds CF, Monk TH, et al. The Pittsburgh Sleep Quality Index: a new instrument for psychiatric practice and research. Psychiatry Res 1989;28:193-213.

9 Fichtenberg NL, Putnam SH, Mann NR, et al. Insomnia screening in postacute traumatic brain injury. Utility and validity of the Pittsburgh Sleep Quality Index. Am J Phys Med Rehabil 2001;80:339-45.

10 Johns M. Reliability and factor analysis of the Epworth Sleepiness Scale. Sleep 1992;15:376-81.

11 Rechtschaffen A, Kales A. A manual of standardized terminology, techniques and scoring for sleep stages of human subjects. Washington, DC: Public Service Publications, U.S. Government Printing Office, 1968.
12 American Academy of Sleep Medicine Task Force. Sleep-related breathing disorders in adults: recommendations for syndrome definition and measurement techniques in clinical research. The Report of an American Academy of Sleep Medicine Task Force. Sleep 1999;22:667-89.

13 Carskadon MA, Dement WC, Mitler MM, et al. Guidelines for the multiple sleep latency test (MSLT): a standard measure of sleepiness. Sleep 1986;9:519-24.

14 Bräutigam C, Wevers RA, Jansen RJT, et al. Biochemical hallmarks of tyrosine hydroxylase deficiency. Clin Chem 1998;44:1897-904.

15 Bonafé L, Thöny B, Leimbacher W, et al. Diagnosis of dopa-responsive dystonia and other tetrahydrobiopterin disorders by the study of biopterin metabolism in fibroblasts. Clin Chem 2001;47:477-85.

16 Romstad A, Dupont E, Krag-Olsen B, et al. Dopa-responsive dystonia and Tourette syndrome in a large Danish family. Arch Neurol 2003;60:618-22.

17 Klein C, Hedrich K, Kabakçi K, et al. Exon deletions in the GCHI gene in two of four Turkish families with dopa-responsive dystonia. Neurology 2002;59:1783-6.

18 The Human Genome Database. http://www.gdb.org (accessed 13 June 2005).

19 Dib C, Faure S, Fizames C, et al. A comprehensive genetic map of the human genome based on 5,264 microsatellites. Nature 1996;380:152-4.

20 Cottingham RW Jr, Idury RM, Schaffer AA. Faster sequential genetic linkage computations. Am J Hum Genet 1993;53:252-63.

21 Steinberger D, Korinthenberg R, Topka H, et al. Dopa-responsive dystonia: mutation analysis of $\mathrm{GCH} 1$ and analysis of therapeutic doses of L-dopa. Neurology 2000;55:1735-7.

$22 \mathrm{Bijl}$ RV, Ravelli A, van Zessen G. Prevalence of psychiatric disorder in the general population: results of the Netherlands Mental Health Survey and Incidence Study (NEMESIS). Soc Psychiatry Psychiatr Epidemiol 1998;33:587-95

23 Kessler RC, Berglund P, Demler O, et al. The epidemiology of major depressive disorder. Results from the National Comorbidity Survey Replication (NCS-R). JAMA 2003;289:3095-105.

24 Angst J. Major depression in 1998: are we providing optimal therapy? J Clin Psychiatry 1999;60(suppl 6):5-9.

25 Fireman B, Koran LM, Leventhal JL, et al. The prevalence of clinically recognized obsessive-compulsive disorder in a large health maintenance organization. Am J Psychiatry 2001; 158:1904-10.

26 Angst J, Gamma A, Endrass J, et al. Obsessive-compulsive severity spectrum in the community: prevalence, comorbidity, and course. Eur Arch Psychiatry Clin Neurosci 2004;254:156-64.

27 Horwath E, Weismann M. The epidemiology and cross-national presentation of obsessive-compulsive disorder. Psychiatr Clin North Am 2000;23:493-506.

28 Grabe HJ, Meyer Ch, Hapke U, et al. Prevalence, quality of life and psychosocial function in obsessive-compulsive disorder in northern Germany. Eur Arch Psychiatry Clin Neurosci 2000;250:262-8.

29 Schäfer D, Greutlich W. Effects of parkinsonian medication on sleep. J Neurol 2000;247(suppl 4):IV/24-27.

30 Segawa M, Nomura Y, Nishiyama N. Autosomal dominant guanosine triphosphate cyclohydrolase I deficiency (Segawa disease). Ann Neurol 2003;54(suppl 6):S32-45.

31 Robinson R, McCarthy GT, Bandmann O, et al. GTP cyclohydrolase deficiency; intrafamilial variation in clinical phenotype, including levodopa responsiveness. J Neurol Neurosurg Psychiatry 1999:66:86-9.

32 Segawa M, Nomura Y, Nishiyama N. Autosomal dominant guanosine triphosphate cyclohydrolase I deficiency (Segawa disease). Ann Neurol 2003;54(suppl 6):S32-45.

33 Mathen D, Marsden CD. SSRI-induced reversal of levodopa benefit in two patients with dopa-responsive dystonia. Mov Disord 1999;14:874-6.

34 Segawa M, Nishiyama N, Nomura Y. DOPA-responsive dystonic parkinsonism - pathophysiologic considerations. In: Stern GM, eds. Parkinson disease. Advances in neurology. Vol 80. Philadelphia: Lippincott, Williams \& Wilkins, 1999:389-400

35 Furukawa Y, Nygaard TG, Gütlich M, et al. Striatal biopterin and tyrosine hydroxylase protein reduction in dopa-responsive dystonia. Neurology 1999:53:1032-41.

36 Garlow S, Musselman L, Nemeroff C. The neurochemistry of mood disorders: clinical studies. In: Charney D, Nestler E, Bunney B, eds. Neurobiology of mental illness. New York: Oxford University Press, 1999:348-64.

37 Duman R. The neurochemistry of mood disorders: preclinical studies. In: Charney D, Nestler E, Bunney B, eds. Neurobiology of mental illness. New York: Oxford University Press, 1999:333-47.

38 Deakin JF. Depression and antisocial personality disorder: two contrasting disorders of 5HT function. J Neural Transm Suppl 2003;64:79-93.

39 Smith KA, Fairburn GG, Cowen PJ. Relapse of depression after rapid depletion of tryptophan. Lancet 1997;349:915-9.

40 Ressler KJ, Nemeroff CB. Role of norepinephrine in the pathophysiology and treatment of mood disorders. Biol Psychiatry 1999;46:1219-33.

41 Anand A, Charney DS. Norepinephrine dysfunction in depression. J Clin Psychiatry 2000;61(suppl 10):16-24.

42 Brunello N, Mendlewicz J, Kasper S, et al. The role of noradrenaline and selective noradrenaline reuptake inhibition in depression. Eur Neuropsychopharmacol 2002;12:461-75.

43 Brotini S, Gigli GL. Epidemiology and clinical features of sleep disorders in extrapyramidal disease. Sleep Med 2004;5:169-79.

44 Jouvet M. Insomnia and decrease of cerebral 5-hydroxytryptamine after destruction of the raphe system in the cat. Adv Pharmacol 1968;6:265-79. 